\title{
Observability of Nonlinear Fractional Dynamical Systems
}

\author{
K. Balachandran, ${ }^{1}$ V. Govindaraj, ${ }^{1}$ M. Rivero, ${ }^{2}$ J. A. Tenreiro Machado, ${ }^{3}$ and J. J. Trujillo ${ }^{4}$ \\ ${ }^{1}$ Department of Mathematics, Bharathiar University, Coimbatore 641 046, India \\ ${ }^{2}$ Department of Electrical Engineering, Institute of Engineering, Polytechnic of Porto, 4200-072 Porto, Portugal \\ ${ }^{3}$ Departamento de Matemática Fundamental, Universidad de La Laguna, La Laguna, 38271 Tenerife, Spain \\ ${ }^{4}$ Departamento de Análisis Matemático, Universidad de La Laguna, La Laguna, 38271 Tenerife, Spain
}

Correspondence should be addressed to J. A. Tenreiro Machado; jtm@isep.ipp.pt

Received 19 March 2013; Accepted 7 June 2013

Academic Editor: Hossein Jafari

Copyright (C) 2013 K. Balachandran et al. This is an open access article distributed under the Creative Commons Attribution License, which permits unrestricted use, distribution, and reproduction in any medium, provided the original work is properly cited.

We study the observability of linear and nonlinear fractional differential systems of order $0<\alpha<1$ by using the Mittag-Leffler matrix function and the application of Banach's contraction mapping theorem. Several examples illustrate the concepts.

\section{Introduction}

Dynamical systems represented by ordinary differential equations are extensively studied in the literature. Many dynamical systems are modeled by fractional differential equations in various fields of science and technology [1-6]. In fact a fractional differential equation is considered as an alternative model to a nonlinear differential equation [7-9]. Recently, fractional differential equations are attracting a large number of researchers which results an increasing number of publications $[10,11]$. Especially, the publications on the theory of fractional differential equations are growing exponentially, but the number of studies on qualitative behaviors of fractional dynamical systems is reduced, and its scope is limited. Therefore, it is important to study the qualitative behavior of fractional dynamical systems.

Observability is one of the structural properties of a fractional dynamical system defined as the possibility to deduce the initial state of the system from observing its inputoutput behavior. Several authors [12-16] have established the results for observability of linear fractional dynamical system of order $0<\alpha<1$ using Gramian matrix and rank conditions. Also the recent monograph [17] reported the same results using rank condition. Motivated by the above discussion, in the present paper we study the observability of linear and nonlinear fractional order systems of order $0<\alpha<1$ with corresponding linear observation using the Mittag-Leffler matrix function and the Banach's contraction mapping theorem. Examples are provided to illustrate the results.

Bearing these ideas in mind, this paper is organized as follows. Section 2 introduces the main fundamental concepts. Sections 3 and 4 analyse the observability of linear and nonlinear systems, respectively. Section 5 presents some examples that illustrate the concepts. Finally, Section 6 outlines the main conclusions.

\section{Preliminaries}

In this section, we introduce the definitions and preliminary results from fractional calculus which are used throughout this paper [18-23].

Definition 1 (see [24]). The Caputo fractional derivative of order $\alpha \in \mathbb{C}$ with $n-1<\alpha \leq n, n \in \mathbb{N}$, for a suitable function $f$, is defined as

$$
\left({ }^{C} D_{0+}^{\alpha} f\right)(t)=\frac{1}{\Gamma(n-\alpha)} \int_{0}^{t}(t-s)^{n-\alpha-1} f^{n}(s) \mathrm{d} s,
$$

where $f^{n}(s)=\mathrm{d}^{n} f / \mathrm{ds}^{\mathrm{n}}$. In particular, if $0<\alpha<1$ then

$$
\left({ }^{C} D_{0+}^{\alpha} f\right)(t)=\frac{1}{\Gamma(1-\alpha)} \int_{0}^{t}(t-s)^{-\alpha} f^{\prime}(s) \mathrm{d} s .
$$

For brevity, the Caputo fractional derivative ${ }^{C} D_{0+}^{\alpha}$ is taken as ${ }^{\mathrm{C}} D^{\alpha}$. 
Definition 2. The Miller-Ross sequential fractional derivative is defined as

$$
\left(D^{k \alpha} f\right)(x)=\left(D^{\alpha} D^{(k-1) \alpha} f\right)(x),
$$

where $k=1, \ldots, n,\left(D^{0} f\right)(x)=f(x)$ and $D^{\alpha}$ is any fractional differential operator, for example, it could be ${ }^{C} D_{a+}^{\alpha}$.

Definition 3 (see [25]). The Mittag-Leffler matrix function for an arbitrary square matrix $A$ is

$$
\begin{gathered}
E_{\alpha, \beta}(A)=\sum_{k=0}^{\infty} \frac{A^{k}}{\Gamma(\alpha k+\beta)}, \quad \alpha, \beta>0, \\
E_{\alpha, 1}(A)=E_{\alpha}(A) \quad \text { with } \beta=1 .
\end{gathered}
$$

Consider the fractional differential equation of order $0<\alpha<1$

$$
\begin{gathered}
{ }^{C} D^{\alpha} x(t)=A x(t)+f(t), \quad t \in[0, T]:=J, \\
x(0)=x_{0},
\end{gathered}
$$

where $A$ is an $n \times n$ matrix and $f(t)$ is a continuous function on $J$. The solution of (5) is given by [26]

$$
\begin{aligned}
x(t)= & E_{\alpha}\left(A t^{\alpha}\right) x_{0} \\
& +\int_{0}^{t}(t-s)^{\alpha-1} E_{\alpha, \alpha}\left(A(t-s)^{\alpha}\right) f(s) \mathrm{d} s .
\end{aligned}
$$

We use this solution representation to study the observability results.

\section{Linear Systems}

Consider the fractional order linear time invariant system

$$
{ }^{C} D^{\alpha} x(t)=A x(t), \quad 0<\alpha<1, t \in J,
$$

with linear observation

$$
y(t)=H x(t),
$$

where $x \in \mathbb{R}^{n}, y \in \mathbb{R}^{m}, A$ is a $n \times n$ matrix, and $H$ is an $m \times n$ matrix.

Definition 4. The system (7) and (8) is observable on an interval $J$ if

$$
y(t)=H x(t)=0, \quad t \in J,
$$

implies that

$$
x(t)=0, \quad t \in J .
$$

Theorem 5. The linear system (7) and (8) is observable on $J$ if and only if the observability Gramian matrix

$$
W(0, T)=\int_{0}^{T} E_{\alpha}\left(A^{*} t^{\alpha}\right) H^{*} H E_{\alpha}\left(A t^{\alpha}\right) \mathrm{d} t
$$

is positive definite.
Proof. The solution $x(t)$ of (7) corresponding to the initial condition $x(0)=x_{0}$ is given by

$$
x(t)=E_{\alpha}\left(A t^{\alpha}\right) x_{0},
$$

and we have, for $y(t)=H x(t)=H E_{\alpha}\left(A t^{\alpha}\right) x_{0}$,

$$
\begin{aligned}
\|y\|^{2} & =\int_{0}^{T} y^{*}(t) y(t) \mathrm{d} t \\
& =x_{0}^{*} \int_{0}^{T} E_{\alpha}\left(A^{*} t^{\alpha}\right) H^{*} H E_{\alpha}\left(A t^{\alpha}\right) \mathrm{d} t x_{0} \\
& =x_{0}^{*} W(0, T) x_{0},
\end{aligned}
$$

a quadratic form in $x_{0}$. Clearly, matrix $W(0, T)$ is $n \times n$ symmetric. If $W(0, T)$ is a positive definite, then $y=0$ implies that $x_{0}^{*} W(0, T) x_{0}=0$. Therefore, it yields that $x_{0}=0$. Hence, the system (7) and (8) is observable on $J$. If $W(0, T)$ is not positive definite, then there is some $x_{0} \neq 0$ such that $x_{0}^{*} W(0, T) x_{0}=0$. Then, $x(t)=E_{\alpha}\left(A t^{\alpha}\right) x_{0} \neq 0$, for $t \in J$, but $\|y\|^{2}=0$, so $y=0$, and we conclude that the system (7) and (8) is not observable on $J$. Hence, the desired result.

If the linear system (7) and (8) is observable on an interval $J$, then $x(0)=x_{0}$, and the initial state, for the solution on that interval, is reconstructed directly from the observation $y(t)=H E_{\alpha}\left(A t^{\alpha}\right) x_{0}$.

Definition 6. The $n \times n$ matrix function $R(t)$ defined on $J$ is an reconstruction kernel if and only if

$$
\int_{0}^{T} R(t) H E_{\alpha}\left(A t^{\alpha}\right) \mathrm{d} t=I .
$$

Theorem 7. There exists a reconstruction kernel $R(t)$ on $J$ if and only if the system (7) and (8) is observable on J.

Proof. If a reconstruction kernel exists and satisfying

$$
\int_{0}^{T} R(t) y(t) \mathrm{d} t=\int_{0}^{T} R(t) H E_{\alpha}\left(A t^{\alpha}\right) \mathrm{d} t x_{0}=x_{0}
$$

and $y(t)=0$, then $x_{0}=0$. So $x(t)=0$, and we conclude that the system (7) and (8) is observable on $J$. If, on the other hand, the system (7) and (8) is observable on $J$, then from Theorem 5

$$
W(0, T)=\int_{0}^{T} E_{\alpha}\left(A^{*} t^{\alpha}\right) H^{*} H E_{\alpha}\left(A t^{\alpha}\right) \mathrm{d} t>0 .
$$

Let

$$
R_{0}(t)=W^{-1}(0, T) E_{\alpha}\left(A^{*} t^{\alpha}\right) H^{*}, \quad t \in J .
$$

Then, we have

$$
\begin{aligned}
& \int_{0}^{T} R_{0}(t) H E_{\alpha}\left(A t^{\alpha}\right) \mathrm{d} t \\
& \quad=W^{-1} \int_{0}^{T} E_{\alpha}\left(A^{*} t^{\alpha}\right) H^{*} H E_{\alpha}\left(A t^{\alpha}\right) \mathrm{d} t=I,
\end{aligned}
$$

so that (17) is a reconstruction kernel on $J$. 


\section{Nonlinear Systems}

Consider the nonlinear system described by the fractional differential equation

$$
{ }^{C} D^{\alpha} x(t)=A x(t)+f(t, x(t)), \quad t \in J,
$$

where $f$ is an $n$ vector and is continuous on $t \in J$, with linear observation

$$
y(t)=H x(t)
$$

where $y$ is an $m$ vector with $m<n$. We assume that the system (19) is observed by the quantity $y$. Then, the problem of observability of (19) is treated as follows: it is required to find the unknown state at the present time $t$, from the quantity $y$ over the interval $[\theta, t]$, where $\theta$ is some past time because, since $m<n$, expression (19) does not allow immediate finding of $x$ and $y$.

Definition 8. The system (19) and (20) is said to be observable at time $t$ if there exists $\theta<t$ such that the state of the system at time $t$ can be identified from knowledge of the system output over the interval $[\theta, t]$. If the system is observable at every $t \epsilon$ $J$, it is called completely observable.

We will assume that (19) has a unique solution for any initial condition. If we take $\tau$ as $\theta<\tau<t$, then the solution of (19) is uniquely defined for $x=x(\tau)$ as the initial condition and is given by

$$
\begin{aligned}
x(t)= & E_{\alpha}\left(A(t-\tau)^{\alpha}\right) x(\tau) \\
& +\int_{\tau}^{t}(t-s)^{\alpha-1} E_{\alpha, \alpha}\left(A(t-s)^{\alpha}\right) f(s, x(s)) \mathrm{d} s .
\end{aligned}
$$

We can rearrange

$x(\tau)$

$$
\begin{aligned}
= & {\left[E_{\alpha}\left(A(t-\tau)^{\alpha}\right)\right]^{-1} } \\
& \times\left[x(t)-\int_{\tau}^{t}(t-s)^{\alpha-1} E_{\alpha, \alpha}\left(A(t-s)^{\alpha}\right) f(s, x(s)) \mathrm{d} s\right],
\end{aligned}
$$

and $y(\tau)$ is given by

$$
\begin{aligned}
& y(\tau) \\
& =\left[E_{\alpha}\left(A(t-\tau)^{\alpha}\right)\right]^{-1} \\
& \times[H x(t)-H \\
& \left.\quad \times \int_{\tau}^{t}(t-s)^{\alpha-1} E_{\alpha, \alpha}\left(A(t-s)^{\alpha}\right) f(s, x(s)) \mathrm{d} s\right],
\end{aligned}
$$

$$
\begin{aligned}
& y(\tau) \\
& =\frac{1}{\left[E_{\alpha}\left(A(t-\tau)^{\alpha}\right)\right]^{2}} \\
& \quad \times[H x(t)-H \\
& \left.\quad \times \int_{\tau}^{t}(t-s)^{\alpha-1} E_{\alpha, \alpha}\left(A(t-s)^{\alpha}\right) f(s, x(s)) \mathrm{d} s\right] \\
& \quad \times E_{\alpha}\left(A(t-\tau)^{\alpha}\right) .
\end{aligned}
$$

Multiplying the above equation by $E_{\alpha}\left(A^{*}(t-\tau)^{\alpha}\right) H^{*}$ from the left and integrating from $\theta$ to $t$, we obtain

$$
\begin{aligned}
& \int_{\theta}^{t}\left[E_{\alpha}\left(A(t-\tau)^{\alpha}\right)\right]^{2} E_{\alpha}\left(A^{*}(t-\tau)^{\alpha}\right) H^{*} y(\tau) \mathrm{d} \tau \\
& =\int_{\theta}^{t} E_{\alpha}\left(A^{*}(t-\tau)^{\alpha}\right) H^{*} H x(t) E_{\alpha}\left(A(t-\tau)^{\alpha}\right) \mathrm{d} \tau \\
& \quad-\int_{\theta}^{t} E_{\alpha}\left(A^{*}(t-\tau)^{\alpha}\right) H^{*} H \\
& \quad \times\left(\int_{\tau}^{t}(t-s)^{\alpha-1} E_{\alpha, \alpha}\left(A(t-s)^{\alpha}\right) f(s, x(s)) \mathrm{d} s\right) \\
& \quad \times E_{\alpha}\left(A(t-\tau)^{\alpha}\right) \mathrm{d} \tau \\
& =\int_{\theta}^{t} E_{\alpha}\left(A^{*}(t-\tau)^{\alpha}\right) H^{*} H E_{\alpha}\left(A(t-\tau)^{\alpha}\right) \mathrm{d} \tau x(t) \\
& \quad-\int_{\theta}^{t}(t-s)^{\alpha-1} E_{\alpha, \alpha}\left(A(t-s)^{\alpha}\right) f(s, x(s)) \\
& \quad \times\left(\int_{\theta}^{s} E_{\alpha}\left(A^{*}(t-\tau)^{\alpha}\right) H^{*} H E_{\alpha}\left(A(t-\tau)^{\alpha}\right) \mathrm{d} \tau\right) \mathrm{d} s \\
& =W(\theta, t) x(t) \\
& \quad \int_{\theta}^{t}(t-s)^{\alpha-1} E_{\alpha, \alpha}\left(A(t-s)^{\alpha}\right) W(\theta, s) f(s, x(s)) \mathrm{d} s .
\end{aligned}
$$

If the matrix $W(\theta, t)$ is invertible, that is, if the truncated linear system (19) and (20) is observable, then, from (24), we have

$$
\begin{aligned}
& x(t)=W^{-1}(\theta, t) \int_{\theta}^{t}[\left.E_{\alpha}\left(A(t-s)^{\alpha}\right)\right]^{2} \\
& \times E_{\alpha}\left(A^{*}(t-s)^{\alpha}\right) H^{*} y(s) \mathrm{d} s \\
&+W^{-1}(\theta, t) \int_{\theta}^{t}(t-s)^{\alpha-1} E_{\alpha, \alpha}\left(A(t-s)^{\alpha}\right) \\
& \\
& \quad \times W(\theta, s) f(s, x(s)) \mathrm{d} s .
\end{aligned}
$$


Now let

$$
\begin{aligned}
& G_{1}(t, \theta, s)= W^{-1}(\theta, t)\left[E_{\alpha}\left(A(t-s)^{\alpha}\right)\right]^{2} \\
& \times E_{\alpha}\left(A^{*}(t-s)^{\alpha}\right) H^{*}, \\
& G_{2}(t, \theta, s)=W^{-1}(\theta, t) E_{\alpha, \alpha}\left(A(t-s)^{\alpha}\right) W(\theta, s) .
\end{aligned}
$$

Then, the following relation is obtained:

$$
\begin{aligned}
x(t)= & \int_{\theta}^{t} G_{1}(t, \theta, s) y(s) \mathrm{d} s \\
& +\int_{\theta}^{t}(t-s)^{\alpha-1} G_{2}(t, \theta, s) f(s, x(s)) \mathrm{d} s .
\end{aligned}
$$

This equation represents the relation of the unknown state $x$ with the observed output $y$ over the interval $[\theta, t]$. Hence, we have the following result.

Theorem 9. The system (19) and (20) is globally (a) observable at $t$ and $(b)$ completely observable, if the following conditions hold.

(i) There exists a constant $c>0$ such that

$$
\operatorname{det}(W(\theta, t)) \geq c
$$

(ii) (27) has a unique solution for any $y$ which is continuous on $[\theta, t](a)$ for some $\theta<t$, in the case of an observable system at time $t$, and $(b)$ for all $t$ and for some $\theta<t$, in the case of $a$ completely observable system.

In (27), time $\theta$ may not be necessarily fixed; therefore, $\theta$ can be replaced by $\tau$. After this change is made, expression (27) is substituted into (22). We obtain that

$$
\begin{aligned}
x(\tau)= & {\left[E_{\alpha}\left(A(t-\tau)^{\alpha}\right)\right]^{-1} } \\
& \times\left[\int_{\tau}^{t} G_{1}(t, \tau, s) y(s) \mathrm{d} s\right. \\
& \quad+\int_{\theta}^{t}(t-s)^{\alpha-1} G_{2}(t, \theta, s) f(s, x(s)) \mathrm{d} s \\
& \left.-\int_{\theta}^{t}(t-s)^{\alpha-1} E_{\alpha, \alpha}\left(A(t-\tau)^{\alpha}\right) f(s, x(s)) \mathrm{d} s\right] \\
= & \int_{\tau}^{t} G_{3}(t, \tau, s) y(s) \mathrm{d} s \\
& +\int_{\tau}^{t}(t-s)^{\alpha-1} G_{4}(t, \tau, s) f(s, x(s)) \mathrm{d} s \quad \text { for } \tau<t
\end{aligned}
$$

where

$$
\begin{aligned}
G_{3}(t, \tau, s)=\left[E_{\alpha}\left(A(t-\tau)^{\alpha}\right)\right]^{-1} G_{1}(t, \tau, s) \\
G_{4}(t, \tau, s)=\left[E_{\alpha}\left(A(t-\tau)^{\alpha}\right)\right]^{-1} \\
\times\left[G_{2}(t, \tau, s)-E_{\alpha, \alpha}\left(A(t-s)^{\alpha}\right)\right] .
\end{aligned}
$$

In Theorem 9, if we replace (27) by (29), the same results are also valid with a simple change of variables. Next, we consider the application of Banach's contraction mapping theorem to these nonlinear equations.

Consider a special system of the form

$$
\begin{gathered}
{ }^{C} D^{\alpha} x(t)=A x(t)+\epsilon f(t, x(t)), \\
y(t)=H x(t),
\end{gathered}
$$

where $\epsilon$ is a scalar positive constant and there exists a constant $k \geq 0$ such that the nonlinear function $f$ satisfies the Lipschitz condition

$$
\left\|f\left(t, x_{1}\right)-f\left(t, x_{2}\right)\right\| \leq k\left\|x_{1}-x_{2}\right\| .
$$

Theorem 10. The system (31) and (32) is globally (a) observable at time $t$ and $(b)$ completely observable, if the following conditions hold.

(i) There exists a constant $c>0$ such that

$$
\operatorname{det}(W(\theta, t)) \geq c
$$

(ii) A positive constant $\epsilon$ satisfies

$$
\epsilon<\frac{\alpha(t-\theta)^{-\alpha}}{k(t, \theta)}
$$

(a) for some $\theta<t$, in the case of an observable system at time $t$, and $(b)$ for all $t$ and for some $\theta<t$, in the case of a completely observable system.

Proof. A general solution $x(t)$ for (31) with initial condition $x=x(\tau)$ is given by

$$
\begin{aligned}
x(\tau) & \\
& =\left[E_{\alpha}\left(A(t-\tau)^{\alpha}\right)\right]^{-1} \\
& \times\left[x(t)-\epsilon \int_{\tau}^{t}(t-s)^{\alpha-1} E_{\alpha, \alpha}\left(A(t-s)^{\alpha}\right) f(s, x(s)) \mathrm{d} s\right] .
\end{aligned}
$$

Just as (27) is derived from (22), the next equation is derived from (36):

$$
\begin{aligned}
x(t)=W^{-1}(\theta, t) \int_{\theta}^{t}\left[E_{\alpha}\left(A(t-s)^{\alpha}\right)\right]^{2} & \\
& \quad \times E_{\alpha}\left(A^{*}(t-s)^{\alpha}\right) H^{*} y(s) \mathrm{d} s \\
+\epsilon W^{-1}(\theta, t) \int_{\theta}^{t}(t-s)^{\alpha-1} E_{\alpha, \alpha}\left(A(t-s)^{\alpha}\right) & \\
& \times W(\theta, s) f(s, x(s)) \mathrm{d} s .
\end{aligned}
$$


Substituting (37) into (36), for every $\tau \in[\theta, t]$, we have

$$
\begin{gathered}
x(\tau)=\left[E_{\alpha}\left(A(t-\tau)^{\alpha}\right)\right]^{-1} \\
\times\left[W^{-1}(\theta, t) \int_{\theta}^{t} E_{\alpha}\left(A^{*}(t-s)^{\alpha}\right)\right. \\
\quad \times H^{*}\left[E_{\alpha}\left(A(t-s)^{\alpha}\right)\right]^{2} y(s) \mathrm{d} s \\
+\epsilon W^{-1}(\theta, t) \int_{\theta}^{t}(t-s)^{\alpha-1} E_{\alpha, \alpha}\left(A(t-s)^{\alpha}\right) \\
\quad \times W(\theta, s) f(s, x(s)) \mathrm{d} s \\
\left.\quad-\epsilon \int_{\tau}^{t}(t-s)^{\alpha-1} E_{\alpha, \alpha}\left(A(t-s)^{\alpha}\right) f(s, x(s)) \mathrm{d} s\right] .
\end{gathered}
$$

Consequently, for the system (31) and (32) to be observable, it is sufficient that the inverse of $W(\theta, t)$ exists, and the solution of (38) exists and is unique. If we assume that there exist solutions $x_{1}, x_{2}$ of (38), for a given $y$ such that $x_{1} \neq x_{2}$, then, using (33), we have

$$
\begin{aligned}
&\left\|x_{1}(\tau)-x_{2}(\tau)\right\| \\
& \leq k \epsilon\left|\left(E_{\alpha}\left(A(t-\tau)^{\alpha}\right)\right)^{-1}\right| \\
& \times \int_{\tau}^{t}(t-s)^{\alpha-1}\left|E_{\alpha, \alpha}\left(A(t-s)^{\alpha}\right)\right|\left|x_{1}-x_{2}\right| \mathrm{d} s \\
&+k \epsilon\left|\left(E_{\alpha}\left(A(t-\tau)^{\alpha}\right)\right)^{-1}\right|\left|W^{-1}(\theta, t)\right| \\
& \times \int_{\theta}^{t}(t-s)^{\alpha-1}\left|E_{\alpha, \alpha}\left(A(t-s)^{\alpha}\right)\right|\left|x_{1}-x_{2}\right| \mathrm{d} s \\
& \leq k \epsilon\left|\left(E_{\alpha}\left(A(t-\tau)^{\alpha}\right)\right)^{-1}\right| \\
& \times \int_{\theta}^{t}(t-s)^{\alpha-1}\left|E_{\alpha, \alpha}\left(A(t-s)^{\alpha}\right)\right|\left|x_{1}-x_{2}\right| \mathrm{d} s \\
&+k \epsilon\left|\left(E_{\alpha}\left(A(t-\tau)^{\alpha}\right)\right)^{-1}\right|\left|W^{-1}(\theta, t)\right| \\
& \times \int_{\theta}^{t}(t-s)^{\alpha-1}\left|E_{\alpha, \alpha}\left(A(t-s)^{\alpha}\right)\right|\left|x_{1}-x_{2}\right| \mathrm{d} s \\
& \leq \frac{\epsilon}{\alpha}(t-\theta)^{\alpha} k_{1}(t, \theta)\left\|x_{1}-x_{2}\right\| \\
&+\frac{\epsilon}{\alpha}(t-\theta)^{\alpha} k_{2}(t, \theta)\left\|x_{1}-x_{2}\right\|,
\end{aligned}
$$

where

$$
\begin{gathered}
k_{1}(t, \theta)=\max _{\theta<\tau<s<t}\left|\left(E_{\alpha}\left(A(t-\tau)^{\alpha}\right)\right)^{-1} E_{\alpha, \alpha}\left(A(t-s)^{\alpha}\right)\right| k, \\
k_{2}(t, \theta)=\max _{\theta<\tau<s<t}\left|\left(E_{\alpha}\left(A(t-\tau)^{\alpha}\right)\right)^{-1} W^{-1}(\theta, t)\right| \\
\quad \times\left|E_{\alpha, \alpha}\left(A(t-s)^{\alpha}\right) W(\theta, t)\right| k .
\end{gathered}
$$

From this, there exists a $k(t, \theta)$ such that

$$
\left\|x_{1}(\tau)-x_{2}(\tau)\right\| \leq \frac{\epsilon}{\alpha} k(t, \theta)(t-\theta)^{\alpha}\left\|x_{1}-x_{2}\right\|
$$

where $k(t, \theta)=k_{1}(t, \theta)+k_{2}(t, \theta)$. If $\epsilon$ satisfies the inequality

$$
\frac{\epsilon}{\alpha} k(t, \theta)(t-\theta)^{\alpha}<1
$$

it follows that $x_{1}=x_{2}$ on $[\theta, t]$. This contradiction leads to the sufficient condition for the observability of system (31) and (32), since the condition (42) obviously guarantees the existence of solutions of (38).

Remark 11. The stability of the fractional system (7) and (19) has been discussed in $[13,27]$. In the case of integer order system, that is, when $\alpha=0,(7)$ becomes an algebraic equation and so $\alpha$ must be greater than zero. When $\alpha=1,(7)$ and (19) become

$$
\begin{gathered}
\dot{x}(t)=A x(t), \\
\dot{x}(t)=A x(t)+f(t, x(t)) .
\end{gathered}
$$

The stability of these systems are related to the eigenvalues of the matrix $A$ and the linear growth condition of the nonlinear function. The solution representation exactly match with the solution of the integer order system, and the stability results are readily follows [28].

\section{Examples}

In this section, we present two examples that illustrate the previous theoretical concepts.

Example 1. Consider that the sequential linear fractional differential equation is

$$
{ }^{C} D_{0+}^{2 \alpha} x(t)+x(t)=0, \quad 0<\alpha<1, t \in[0,1] .
$$

Let us introduce the auxiliary variables $x_{1}(t)=x(t)$ and $x_{2}(t)={ }^{C} D_{0+}^{\alpha} x_{1}(t)$. Then,

$$
\begin{aligned}
& { }^{C} D_{0+}^{\alpha} x_{1}(t)={ }^{C} D_{0+}^{\alpha} x(t)=x_{2}(t), \\
& { }^{C} D_{0+}^{\alpha} x_{2}(t)={ }^{C} D_{0+}^{2 \alpha} x(t)=-x_{1}(t),
\end{aligned}
$$

and, therefore, problem (44) can be expressed as ${ }^{C} D_{0+}^{\alpha} \bar{x}(t)=$ $A \bar{x}(t)$, where $A=\left[\begin{array}{cc}0 & 1 \\ -1 & 0\end{array}\right]$ and $\bar{x}(t)=\left[\begin{array}{l}x_{1}(t) \\ x_{2}(t)\end{array}\right]$. Suppose that observation for the system (44) is $y(t)=x_{1}(t)=$ $(1 / 2) e^{-t}+(1 / 2) e^{-t} \operatorname{erfi}(\sqrt{t})$. Let us take $\alpha=1 / 2$. We pose the problem of computing $x_{1}(0)$ and $x_{2}(0)$. The Mittag-Leffler matrix function, for the given matrix $A$, is given by

$$
E_{1 / 2}\left(A t^{1 / 2}\right)=\left(\begin{array}{cc}
e^{-t} & e^{-t} \operatorname{erfi}(\sqrt{t}) \\
e^{-t} \operatorname{erfi}(\sqrt{t}) & e^{-t}
\end{array}\right)
$$


The observability Gramian matrix for this system is

$$
\begin{aligned}
W(0,1) & =\int_{0}^{1} E_{1 / 2}\left(A^{*} t^{1 / 2}\right) H^{*} H E_{1 / 2}\left(A t^{1 / 2}\right) \mathrm{d} t \\
& =\int_{0}^{1}\left(\begin{array}{cc}
e^{-2 t} & e^{-2 t} \operatorname{erfi}(\sqrt{t}) \\
e^{-2 t} \operatorname{erfi}(\sqrt{t}) & e^{-2 t} \operatorname{erfi}(\sqrt{t})^{2}
\end{array}\right) \mathrm{d} t \\
& =\left(\begin{array}{ll}
0.432332 & 0.309670 \\
0.309670 & 0.286423
\end{array}\right) .
\end{aligned}
$$

Here, $W(0,1)$ is nonsingular, and then its inverse exists:

$$
W^{-1}(0,1)=\left(\begin{array}{cc}
10.2535 & -11.0857 \\
-11.0857 & 15.4768
\end{array}\right) \text {. }
$$

The reconstruction formula gives

$$
\begin{aligned}
\bar{x}(0)= & \int_{0}^{1} R(t) y(t) \mathrm{d} t \\
= & \int_{0}^{1} W^{-1}(0,1) E_{1 / 2}\left(A^{*} t^{1 / 2}\right) H^{*} y(t) \mathrm{d} t \\
= & \frac{1}{2} \int_{0}^{1}\left(\begin{array}{c}
10.2535 e^{-1}-11.0857 e^{-t} \operatorname{erfi}(\sqrt{t}) \\
-11.0857 e^{-1}+15.4768 e^{-t} \operatorname{erfi}(\sqrt{t})
\end{array}\right) \\
& \times\left(e^{-t}+e^{-t} \operatorname{erfi}(\sqrt{t})\right) \mathrm{d} t \\
= & \left(\begin{array}{c}
0.500007 \\
0.499998
\end{array}\right) .
\end{aligned}
$$

Then, we conclude that $x_{1}(0)=0.500007$ and $x_{2}(0)=$ 0.499998 .

Example 2. Consider that the sequential nonlinear fractional differential equation is

$$
{ }^{C} D_{0+}^{2 \alpha} x(t)-x(t)=\frac{1}{x^{2}+1}, \quad 0<\alpha<1, t \in[0,2] .
$$

Let us introduce the following auxiliary variables $x_{1}(t)=x(t)$ and $x_{2}(t)={ }^{C} D_{0+}^{\alpha} x_{1}(t)$. Then,

$$
\begin{gathered}
{ }^{C} D_{0+}^{\alpha} x_{1}(t)={ }^{C} D_{0+}^{\alpha} x(t)=x_{2}(t) \\
{ }^{C} D_{0+}^{\alpha} x_{2}(t)={ }^{C} D_{0+}^{2 \alpha} x(t)=x_{1}(t)+\frac{1}{x_{1}^{2}+1} .
\end{gathered}
$$

Therefore, problem (50) can be expressed as ${ }^{C} D_{0+}^{\alpha} \bar{x}(t)=$ $A \bar{x}(t)+f(t, \bar{x}(t))$, where $A=\left[\begin{array}{ll}0 & 1 \\ 1 & 0\end{array}\right], f(t, \bar{x}(t))=\left(\begin{array}{c}0 \\ 1 /\left(x_{1}^{2}+1\right)\end{array}\right)$ and $\bar{x}(t)=\left[\begin{array}{l}x_{1}(t) \\ x_{2}(t)\end{array}\right]$. Suppose that observation for the system (50) is $y(t)=x_{2}(t)$. Let us take $\alpha=3 / 4$. The Mittag-Leffler matrix function for the given matrix $A$ is

$$
E_{3 / 4}\left(A t^{3 / 4}\right)=\left(\begin{array}{ll}
N_{1}(t) & N_{2}(t) \\
N_{2}(t) & N_{1}(t)
\end{array}\right),
$$

where $N_{1}(t)=(1 / 2)\left[E_{3 / 4}\left(t^{3 / 4}\right)+E_{3 / 4}\left((-t)^{3 / 4}\right)\right]$ and $N_{2}(t)=$ $(1 / 2)\left[E_{3 / 4}\left(t^{3 / 4}\right)-E_{3 / 4}\left((-t)^{3 / 4}\right)\right]$. The observability Gramian matrix for this system,

$$
\begin{aligned}
& W(0,2)=\int_{0}^{2} E_{3 / 4}\left(A^{*} t^{3 / 4}\right) H^{*} H E_{3 / 4}\left(A t^{3 / 4}\right) \mathrm{d} t \\
& =\int_{0}^{2}\left(\begin{array}{cc}
N_{2}^{2}(t) & N_{1}(t) N_{2}(t) \\
N_{1}(t) N_{2}(t) & N_{1}^{2}(t)
\end{array}\right) \mathrm{d} t \\
& =\left(\begin{array}{cc}
10.006 & 11.2441 \\
11.2441 & 12.9504
\end{array}\right) \text {, }
\end{aligned}
$$

is positive definite. Then, $W^{-1}(0,2)$ exists, and the nonlinear function $f(t, \bar{x}(t))$ satisfies the condition (33) with constant $k=2$. By the application of Banach's contraction mapping theorem, the solution of this system exists and is unique. By Theorem 10, the system (50) is globally (a) observable at time $t$ and $(b)$ completely observable.

A final remark noting that $x_{2}$ is not a state variable in the classical case, but maybe we could consider it as a certain "pseudostate variable," without any real interpretation till the moment.

\section{Conclusions}

In this paper, we reviewed the main concepts underlying the observability of linear and nonlinear fractional differential systems of order $0<\alpha<1$. We considered the Mittag-Leffler matrix function and the application of Banach's contraction mapping theorem. Two examples were analyzed illustrating the observability concepts.

\section{Acknowledgment}

This work is partly supported by Project MTM2010-16499 from the Government of Spain and FEDER from EU.

\section{References}

[1] J. A. T. Machado, "And i say to myself: what a fractional world!," Fractional Calculus and Applied Analysis, vol. 14, no. 4, pp. 635654, 2011.

[2] R. L. Magin, Fractional Calculus in Bioengineering, Begell House, Redding, Mass, USA, 2006.

[3] I. Petráš, Fractional-Order Nonlinear Systems: Modeling, Analysis and Simulation, Springer, Heidelberg, Germany, 2011.

[4] V. E. Tarasov, Fractional Dynamics: Applications of Fractional Calculus to Dynamics of Particles, Fields and Media, Nonlinear Physical Science, Springer, Heidelberg, Germany, 2010.

[5] D. Valério and J. S. da Costa, An Introduction to Fractional Control, IET, Stevenage, UK, 2012.

[6] G. M. Zaslavsky, Hamiltonian Chaos and Fractional Dynamics, Oxford University Press, Oxford, UK, 2008.

[7] B. Bonilla, M. Rivero, L. Rodríguez-Germá, and J. J. Trujillo, "Fractional differential equations as alternative models to nonlinear differential equations," Applied Mathematics and Computation, vol. 187, no. 1, pp. 79-88, 2007. 
[8] M. Klimek, On Solutions of Linear Fractional Differential Equations of a Variational Type, Czestochowa University of Technology, Czestochowa, Poland, 2009.

[9] V. V. Uchaikin, Fractional Derivatives for Physicists and Engineers, Volume 1. Back-Ground and Theory; Volume II. Applications, Springer Jointly published with Higher Education Press, 2013.

[10] J. T. Machado, V. Kiryakova, and F. Mainardi, "Recent history of fractional calculus," Communications in Nonlinear Science and Numerical Simulation, vol. 16, no. 3, pp. 1140-1153, 2011.

[11] J. Tenreiro Machado, A. M. Galhano, and J. J. Trujillo, "Science metrics on fractional calculus development since 1966," Fractional Calculus and Applied Analysis, vol. 16, no. 2, pp. 479-500, 2013.

[12] M. Bettayeb and S. Djennoune, "New results on the controllability and observability of fractional dynamical systems," Journal of Vibration and Control, vol. 14, no. 9-10, pp. 1531-1541, 2008.

[13] D. Matignon and B. d'Andréa-Novel, "Some results on controllability and observability of finite dimensional fractional differential systems," in Proceedings of the IAMCS, IEEE Conference on Systems, Man and Cybernetics, pp. 952-956, Lille, France, 1996.

[14] J. Sabatier, M. Merveillaut, L. Fenetau, and A. Oustaloup, "On observability of fractional order systems," in Proceedings of the ASME International Design Engineering Technical Conferences and Computers and Information in Engineering Conference (DETC '09), pp. 253-260, September 2009.

[15] A. B. Shamardan and M. R. A. Moubarak, "Controllability and observability for fractional control systems," Journal of Fractional Calculus, vol. 15, pp. 25-34, 1999.

[16] B. M. Vinagre, C. A. Monje, and A. J. Calderon, "Fractional order systems and fractional order control actions," Fractional Calculus Applications in Automatic Control and Robotics, 2002.

[17] C. A. Monje, Y. Chen, B. M. Vinagre, D. Xue, and V. Feliu, Fractional-Order Systems and Controls, Springer, London, UK, 2010.

[18] D. Baleanu, K. Diethelm, E. Scalas, and J. J. Trujillo, Fractional Calculus. Models and Numerical Methods, vol. 3, World Scientific Publishing, Hackensack, NJ, USA, 2012.

[19] K. Diethelm, The Analysis of Fractional Differential Equations, vol. 2004, Springer, Berlin, Germany, 2010.

[20] A. A. Kilbas, H. M. Srivastava, and J. J. Trujillo, Theory and Applications of Fractional Differential Equations, vol. 204, Elsevier Science B.V., Amsterdam, The Netherlands, 2006.

[21] F. Mainardi and R. Gorenflo, "On Mittag-Leffler-type functions in fractional evolution processes," Journal of Computational and Applied Mathematics, vol. 118, no. 1-2, pp. 283-299, 2000.

[22] K. S. Miller and B. Ross, An Introduction to the Fractional Calculus and Fractional Differential Equations, John Wiley \& Sons Inc., New York, NY, USA, 1993.

[23] K. B. Oldham and J. Spanier, The Fractional Calculus, Academic Press, London, UK, 1974.

[24] M. Caputo, "Linear model of dissipation whose $Q$ is almost frequency independent," Geophysical Journal of Royal Astronomical Society, vol. 13, pp. 529-539, 1967.

[25] S. D. Éidel'man and A. A. Chikriǔ, "Dynamic game-theoretic approach problems for fractional-order equations," Ukrainian Mathematical Journal, vol. 52, no. 11, pp. 1787-1806, 2000.

[26] T. Kaczorek, Selected Problems of Fractional Systems Theory, vol. 411, Springer, Berlin, Germany, 2011.

[27] X.-J. Wen, Z.-M. Wu, and J.-G. Lu, "Stability analysis of a class of nonlinear fractional-order systems," IEEE Transactions on Circuits and Systems II, vol. 55, no. 11, pp. 1178-1182, 2008.
[28] J. Zabczyk, Mathematical Control Theory: An Introduction, Birkhäuser, Berlin, Germany, 1992. 


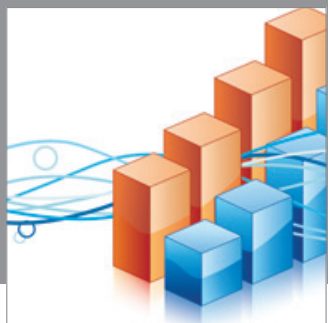

Advances in

Operations Research

mansans

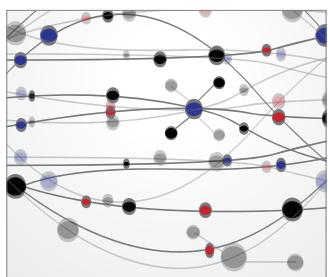

The Scientific World Journal

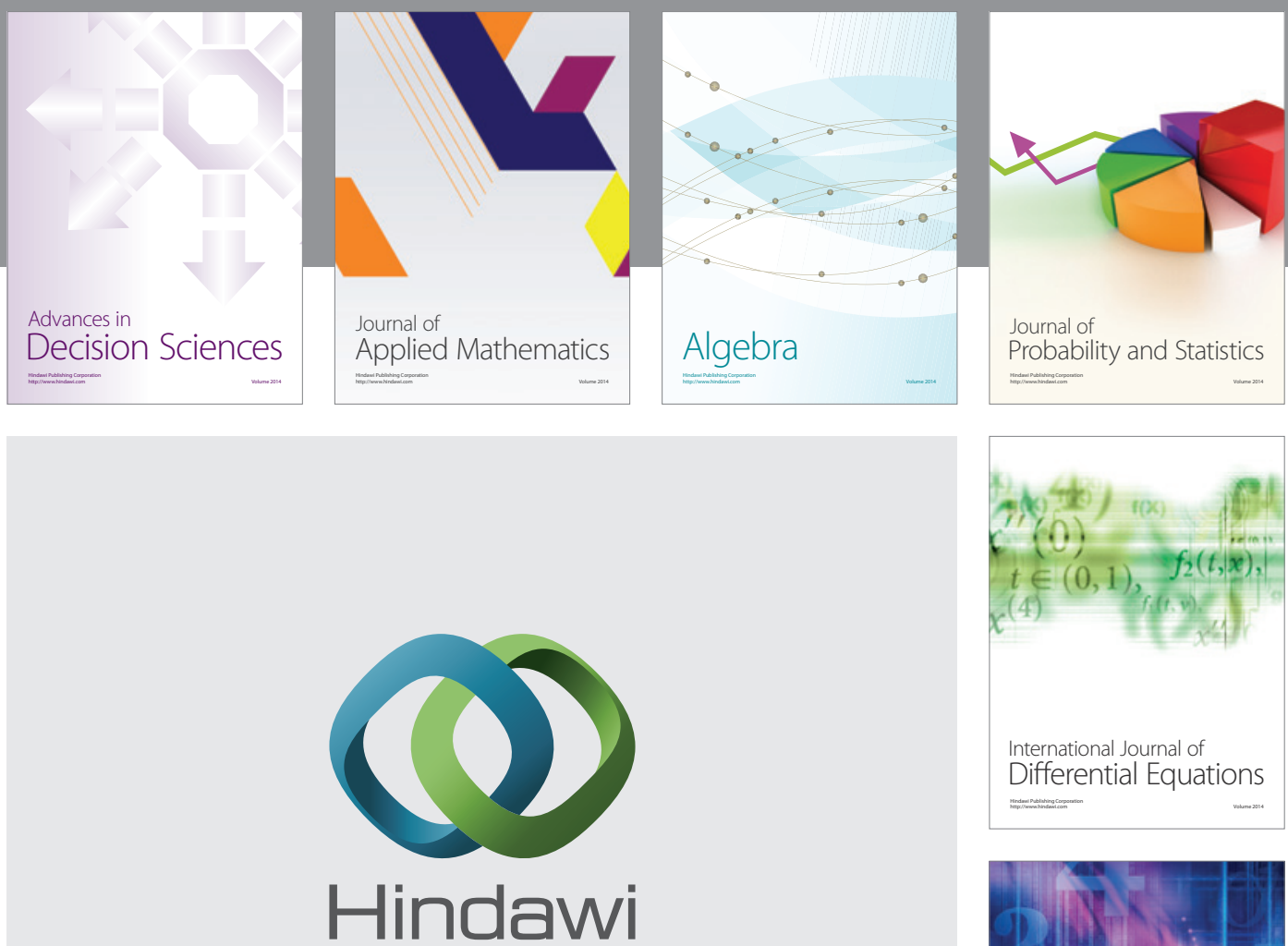

Submit your manuscripts at http://www.hindawi.com


Journal of

Function Spaces

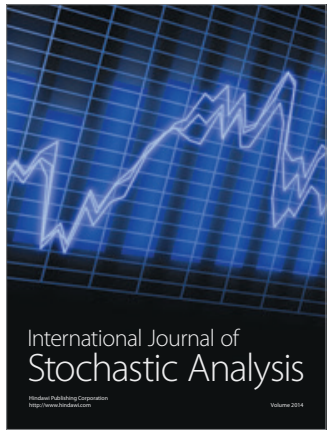

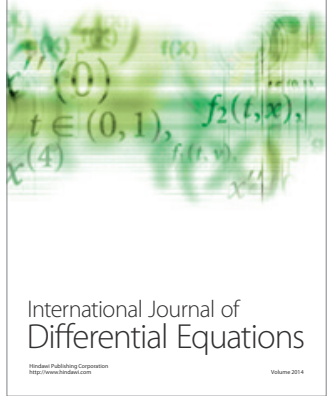
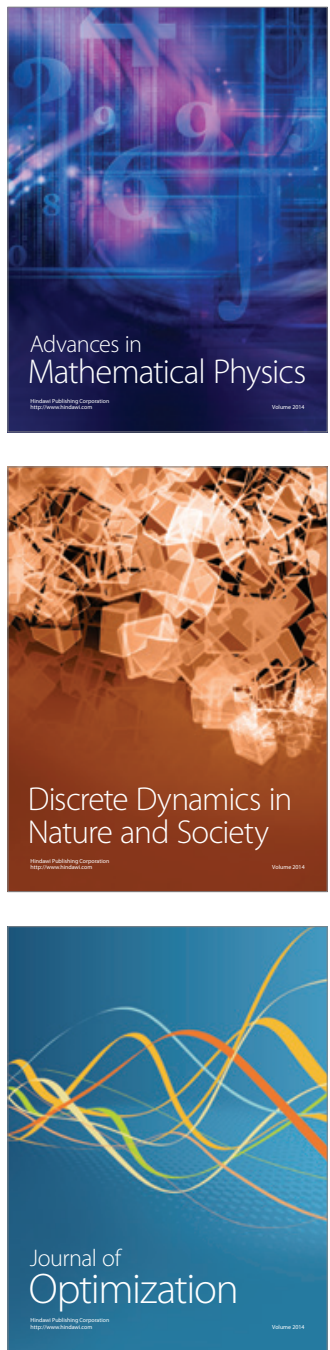